\title{
Added benefits of early detection of other diseases on low-dose CT screening
}

\author{
Rowena Yip ${ }^{1}$, Artit Jirapatnakul ${ }^{1}$, Minxia $\mathrm{Hu}^{2}$, Xiangmeng Chen ${ }^{3}$, Dan $\mathrm{Han}^{4}$, Teng $\mathrm{Ma}^{5}$, Yeqing $\mathrm{Zhu}^{1}$, \\ Mary M. Salvatore ${ }^{6}$, Laurie R. Margolies ${ }^{1}$, David F. Yankelevitz ${ }^{1}$, Claudia I. Henschke ${ }^{1}$; for the I-ELCAP \\ Investigators
}

${ }^{1}$ Department of Radiology, Icahn School of Medicine at Mount Sinai, New York, NY, USA; ${ }^{2}$ Department of Diagnostic Ultrasound, Beijing Tongren Hospital, Capital Medical University, Beijing, China; ${ }^{3}$ Department of Radiology, Jiangmen Central Hospital, Jiangmen, China; ${ }^{4}$ Department of Radiology, Beijing Friendship Hospital, Capital Medical University, Xicheng District, Beijing, China; ${ }^{5}$ Department of Radiology, Tong Ren Hospital, Capital Medical University, Beijing, China; ${ }^{6}$ Department of Radiology, Columbia University Medical Center, New York, NY, USA

Contributions: (I) Conception and design: CI Henschke, DF Yankelevitz, R Yip, A Jirapatnakul; (II) Administrative support: CI Henschke, DF Yankelevitz, R Yip, A Jirapatnakul; (III) Provision of study materials or patients: CI Henschke, DF Yankelevitz, R Yip, A Jirapatnakul; (IV) Collection and assembly of data: All authors; (V) Data analysis and interpretation: All authors; (VI) Manuscript writing: All authors; (VII) Final approval of manuscript: All authors.

Correspondence to: Claudia I. Henschke, PhD, MD. Department of Radiology, Icahn School of Medicine at Mount Sinai, One Gustave L. Levy Place, New York, NY 10029, USA. Email: claudia.henschke@mountsinai.org.

\begin{abstract}
Low-dose CT screening for lung cancer provides images of the entire chest and upper abdomen. While the focus of screening is on finding early lung cancer, radiology leadership has embraced the fact that the information contained in the images presents a new challenge to the radiology profession. Other findings in the chest and upper abdomen were not the reason for obtaining the screening CT scan, nor symptomprompted, but still need to be reported. Reporting these findings and making recommendations for further workup requires careful consideration to avoid unnecessary workup or interventions while still maximizing the benefit that early identification of these other diseases provided. Other potential findings, such as cardiovascular disease and chronic pulmonary obstructive diseases actually cause more deaths than lung cancer. Existing recommendations for workup of abnormal CT findings are based on symptom-prompted indications for imaging. These recommendations may be different when the abnormalities are identified in asymptomatic people undergoing CT screening for lung cancer. I-ELCAP, a large prospectively collected multi-institutional and multi-national database of screenings, was used to analyze CT findings identified in screening for lung cancer. These analyses and recommendations were made by radiologists in collaboration with clinicians in different medical specialties.
\end{abstract}

Keywords: Health check; ancillary findings; lung cancer; chronic obstructive pulmonary disease (COPD); cardiovascular disease; breast

Submitted Jun 16, 2020. Accepted for publication Nov 25, 2020.

doi: $10.21037 /$ tlcr-20-746

View this article at: http://dx.doi.org/10.21037/tlcr-20-746

\section{Introduction}

Low-dose CT (LDCT) screening is approved for reimbursement by insurance companies and the Centers for Medicare and Medicaid Services (CMS) in the United States since 2015 (1). A study sponsored by the National Institutes of Health and The Bill and Melinda Gates Foundation found that between 1990 and 2016, ischemic heart disease, lung cancer, and chronic obstructive pulmonary disease (COPD) are the top three killers in the United States (2) and also worldwide (3).

LDCT, while screening for lung cancer, also 
provides information on asymptomatic people who have unrecognized ischemic heart disease $(4,5)$ and COPD $(6,7)$, both killing more people than lung cancer each year.

Recognition of unsuspected diseases on imaging tests performed for different indications led to an editorial by Lee and Forman in Radiology (8) in 2010 regarding three articles which demonstrated that useful, but unsolicited, quantitative information could be obtained from CT scans about coronary artery calcifications (CAC) (5), bone density (9), and the size of the aorta (10). In their editorial, Lee and Forman reached the following conclusion: "This paradigm shift allows for a rich avenue of further research and development. Rather than shying away from this new responsibility, the radiology leadership should embrace the possibility of adding a new dimension to our profession. By extracting potentially important information from existing images beyond our usual interpretation, we as radiologists can cement the three tenets that define our specialty: our mastery of technology, our clinical acumen, and our dedication to patient safety and quality."

Cardiovascular disease and pulmonary diseases have been identified in a significant proportion of people, being screened or being imaged for other reasons, who are unaware of their disease $(4-7,11)$. This approach has now been incorporated in LDCT screening for lung cancer in regard to CAC; the American College of Radiology registry includes this as a required data element $(12,13)$.

LDCT is a low-radiation dose scan without contrast injection that requires only seconds to obtain and it can be used to identify early manifestations of three of the top ten causes of death. Beyond these diseases, the LDCT provides information on other diseases of the lungs, mediastinum, breasts, bones, and upper abdomen, which may be better treated when identified early. The current vision of LDCT screening provides a comprehensive "health check" of the lungs, heart, and other organs visualized on the LDCT, particularly as LDCT radiation doses are almost as low as chest radiography (14-17). LDCT findings and follow-up recommendations have been developed over the past 20 years of LDCT screening since the initial publication on LDCT screening and long-term follow-up (18-20). There is consensus that relevant findings of these other organs are important components of the LDCT report provided to each screening participant.

A comprehensive "health check" optimal LDCT screening requires a carefully-specified, validated regimen for identification and interpretation of critical LDCT findings and the appropriate follow-up recommendations. Once developed, the recommendations have been incorporated into the International Early Lung Cancer Action Program (I-ELCAP) screening protocol (21) which has allowed for further evaluation of the usefulness of the recommendations. This vision is gaining increasing recognition throughout the world. An entire session at the $20^{\text {th }}$ World Conference on Lung Cancer (WCLC) in Barcelona, Spain in September 2019 was devoted to these other findings (22).

Structured reporting of LDCT screenings and a comprehensive screening management system has allowed for the development of the recommendations. In addition, a common CT acquisition and image reconstruction protocol for both baseline and repeat screenings is important for the interpretation of the findings and change over time, particularly also for future advanced image analytics. Of course, over time, these protocols need to be updated with understanding that this can impact the interpretations.

The resulting large, well-documented database has also enabled the advancement of image analytics and statistical techniques for computer-aided diagnosis of diseases that can be identified in screening. For cardiovascular disease, automated image analysis software can measure the amount of CAC accurately, with a correlation coefficient of 0.88 compared to visual scoring (23); deep-learning approaches have also shown good performance for measuring CAC (24-26). There are also automated techniques for measuring the pulmonary artery and aorta that show good agreement with manual segmentation, with a Dice similarity coefficient of 0.933 (27). Automated image analytics have also shown promise for detecting pulmonary diseases such as emphysema (28-31), airway wall thickening $(32,33)$, all seen in COPD $(34,35)$, and interstitial lung disease (ILD) $(36,37)$. In these analyses, the lung parenchyma is classified according to density and texture using machine learning and deep learning methods. An automated algorithm was able to identify early stage usual interstitial pneumonia (UIP), a form of ILD, with an area under the receiver operating characteristic curve of 0.95 (36). In addition to pulmonary and cardiovascular disease, from the same screening LDCT, automated image analytic algorithms have been used to measure breast density (38), cardiac visceral fat (39), and liver density (40). Low liver density is associated with hepatic steatosis, and the automated image analytic technique was well correlated with manual measurements by a radiologist, with an intraclass correlation coefficient of 0.94 (40). These algorithms are in various stages of development; many have been integrated into commercial systems, but often as part of separate packages that require 
extensive radiologist interaction.

In the future, as algorithms continue to improve, assessments can be made quantitatively and automatically immediately upon the LDCT screening being obtained. These would immediately be included in the radiologic report (41). Currently, however, the CT findings must be identified and measured by radiologists.

Below we briefly discuss each of the added findings that can be identified on LDCT screening for lung cancer, other than lung nodules. The protocol for lung cancer screening and identification of potential lung cancers has been extensively reported, including in our recent article (41). In this report, we focus on the key findings and recommendations for the added findings that we have developed. A summary of each of the findings and recommendations are summarized in Appendix 1. Other important findings, such as osteoporosis leading to subsequent fractures and bronchiectasis are frequent finding in participants in a screening program, and early diagnosis may avoid subsequent significant morbidities, particularly in those participants with COPD. These are being currently being addressed but are not yet completed.

The 2016 Society of Cardiovascular Computed Tomography and the Society of Thoracic Radiology (SCCT/STR) guidelines on non-contrast, non-gated chest CT scans provide a comprehensive review of the best scientific evidence and practice patterns of experts with practical recommendations based on CAC scoring methodologies, interpretation and reporting (13). The guidelines state that $\mathrm{CAC}$ should be evaluated and reported on all non-contrast chest CT examinations (Class I indication). They also suggested that CAC should be estimated as none, mild, moderate or severe (Class I), that it is reasonable to perform ordinal assessment of CAC which assigns a score of 0 to 12 (Class IIa) (see Appendix 1) or to perform Agatston CAC scoring (Class IIb) on all noncontrast chest CT examinations. The prognostic value of these measuring methods for cardiovascular events has been well documented.

\section{Coronary artery calcifications (CAC)}

CAC can be easily detected, measured, and reported on lung screening CT without extra radiation or cost. Reporting on CAC enhances the benefit of lung cancer screening by providing the clinicians with an additional powerful risk stratification tool that can improve the management of primary prevention of cardiovascular events particularly for the patient-clinician's discussion regarding the initiation/ withhold/intensification/avoidance of statin treatment for primary cardiovascular disease prevention as CAC is well accepted as a potent CVD risk modifier.

\section{Aortic valve calcifications (AVC)}

AVC measured on electro-cardiographic-gated (ECG) CT using Agatston score, has been shown to be of prognostic importance for future CVD events, cardiovascular death, and all-cause mortality in the general population $(42,43)$. It is of particular importance in patients with diabetes, aortic stenosis, or on hemodialysis. Meta-analyses also demonstrated the prognostic significance of $\operatorname{AVC}(44,45)$.

AVC on LDCT has been shown to predict death from CVD in smokers beyond that provided by CAC $(46,47)$. LDCT scans can be used to classify AVC as none (AVC score $=0$ ), mild (AVC score $=1$ ), moderate (AVC score $=2$ ), and severe (AVC score $=3$ ) and provide recommendations for further cardiac evaluation (47) (Appendix 1). Review of a cohort of 8,618 smokers enrolled in LDCT screening for lung cancer in New York State between June 2000 and December 2005 showed that the prevalence of AVC significantly increased $(\mathrm{P}<0.0001)$ with the increasing severity of the Ordinal CAC scores (36). CAC and AVC were significant predictors of CVD death when considered alone using multivariable Cox regression analysis (adjusted $\mathrm{HR}$ of $\mathrm{CAC}=1.57, \mathrm{P}=0.04$; adjusted $\mathrm{HR}$ of $\mathrm{AVC}=1.39$, $\mathrm{P}=0.045)$. For $\mathrm{AVC}>0$ and $\mathrm{CAC} \geq 4$, the hazard ratio of CVD death was 2.35 (95\% CI: 1.57-3.50) compared with the reference group of $\mathrm{AVC}=0$ and $\mathrm{CAC}<4$, when adjusted for other risk factors. As the presence of AVC identified on LDCT is a significant predictor of future CVD death, particularly for those with ordinal CAC score $\geq 4$, AVC scores should also be reported on screening LDCTs. For moderate and severe AVC, referral to a cardiologist is recommended and possible echocardiography as there is a high probability of aortic stenosis.

\section{Pulmonary bypertension}

Pulmonary hypertension is a progressive, potentially fatal disease, it is often difficult to diagnose early due to nonspecific nature of symptoms. Pulmonary hypertension is associated with increased morbidity and death in many respiratory and cardiac disorders, and with all-cause mortality, independent of age and cardiopulmonary disease (48-53). The main pulmonary artery diameter (MPA), and 


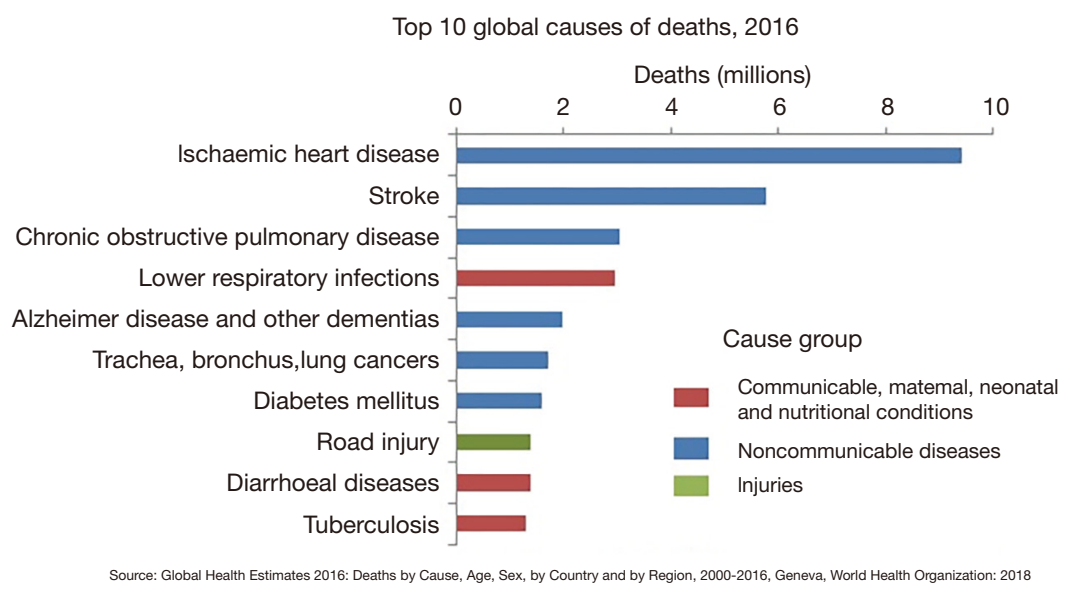

Figure 1 Most frequent causes of death.

ratio of MPA to adjacent ascending aorta (AA), MPA:AA, on Chest CT are strong indicators of suspected pulmonary hypertension.

We reviewed consecutive baseline LDCT scans of 1949 smokers in an Institutional Review Board (IRB)-approved study (54). We measured the diameter of the MPA and AA on an axial CT image at the level of the MPA bifurcation at the widest diameter vertical to its long axis and of the adjacent AA diameter by experienced chest radiologists in the smokers participating in the screening program. We determined the mean and standard deviation of the MPA and the MPA:AA ratio. Abnormally high values were defined as being more than two standard deviations above the mean which was $\mathrm{MPA} \geq 34 \mathrm{~mm}$ and MPA:AA $\geq 1.0$. The prevalence of MPA $\geq 34 \mathrm{~mm}$ and MPA:AA $\geq 1.0$ was $4.2 \%$ and $6.9 \%$, respectively. Multivariable regression demonstrated that body mass index was a significant risk factor, both for MPA $\geq 34 \mathrm{~mm}(\mathrm{OR}=1.07, \mathrm{P}<0.0001)$ and $\mathrm{MPA}: \mathrm{AA} \geq 1.0(\mathrm{OR}=1.04$, $\mathrm{P}=0.003)$. Emphysema was significant in the univariate but not in the multivariate analysis. If pulmonary hypertension is confirmed, its natural history may be improved following targeted therapy specific for the different possible etiologies (54).

In view of the significant risk of morbidity and mortality in subjects with pulmonary hypertension, an MPA $\geq 34 \mathrm{~mm}$ or MPA:AA $\geq 1.0$ should prompt a pulmonary consult to determine its etiology, and an evaluation of symptoms, signs, or illnesses associated with pulmonary hypertension. If it is confirmed, the natural history of pulmonary hypertension may be improved following targeted therapy specific for the different possible etiologies (54).

\section{Pulmonary diseases}

\section{Chronic obstructive pulmonary disease (COPD)}

COPD can be detected on LDCT screening for lung cancer. As shown in Figure 1, COPD is a leading cause of death and causes more deaths than lung cancer. COPD is characterized by chronic airflow limitation caused by a mixture of small airways disease and parenchymal destruction (emphysema) (6,7). Until CT was available, pathologic diagnosis of emphysema was made either after a lung resection or after death. Alterations in the diffusion capacity for carbon monoxide (DLCO), measured in the pulmonary function laboratory, can indicate the presence of emphysema. With the advent of computed tomography, emphysema can now be diagnosed and quantified noninvasively. In 2007, de Torres and his colleagues (6) were the first to show that the presence of emphysema on LDCT was associated with a 2.5 -fold increased risk of lung cancer. Interestingly, de Torres et al showed that the presence of COPD defined by spirometric criteria (i.e., FEV1/FVC $<70 \%$ ) was associated with an increased risk of lung cancer in a univariate analysis, but no longer in the multivariable analysis after adjusting for the presence of emphysema while emphysema remained an independent predictor of lung cancer risk. These results have been confirmed and validated by subsequent studies which also demonstrated that emphysema and COPD are very prevalent, but underdiagnosed (11). Lung cancer screening provides an opportunity to uncover the high proportion of individuals with emphysema and abnormal airway thickening who have never been diagnosed. 
Data showed that $80 \%$ of individuals with lung cancer will have COPD, emphysema or both (6). It is very likely that the improvements in all-cause mortality observed in several randomized controlled studies (55) and the longterm survival rates observed in one-armed studies such as I-ELCAP (19) reflect in part the benefit of earlier diagnoses of COPD and/or emphysema. In other words, lung cancer screening may be the first intervention that actually improves mortality rates in patients with COPD.

\section{Pulmonary fibrosis}

Pulmonary fibrosis is scarring of the lung parenchyma. There are many types of pulmonary fibrosis but idiopathic pulmonary fibrosis (IPF) is the most common in the United States and has the worst prognosis with median survival ranging from 2 to 5 years $(50,51)$.

IPF is a progressive fibrosis disease without known etiology affecting older men and women.

In order to make the diagnosis of IPF, a patient must have no known cause for their fibrosis and have a UIP pattern on chest CT. If the findings on chest CT are inconclusive, the patient may need a biopsy for diagnosis, which is associated with risk of fibrosis exacerbation (56-59). LDCT scans used for lung cancer screening are useful for diagnosing fibrosis.

IPF is associated with cigarette smoking and older age. Therefore, it is not surprising that when we reviewed 951 lung screening participants, that 63 (6.6\%) had pulmonary fibrosis (56), much higher than reported in the general population which is 30 among 100,000 people. IPF was significantly more frequent in men $(\mathrm{P}=0.007)$ and associated with increasing age $(\mathrm{P}<0.0001)$. The most common pattern was peripheral fibrosis in multiple lobes without honeycombing. The presence of honeycombing was significantly associated with progression of fibrosis $(\mathrm{P}=0.0001)$ and extent $(\mathrm{P}=0.005)$. Early diagnosis is important as better treatments that delay progression have been developed.

A radiologist can make the confident diagnosis of UIP on high resolution CT when a patient has sub pleural basilar predominant fibrosis and honeycombing as described by the American Thoracic Society (ATS) guidelines (57). Honeycombing is defined by stacked cysts which touch the pleural surface; it typically represents advanced disease. If there is no honeycombing, then the radiologists should call it a "Probable UIP" pattern and biopsy may be necessary for confirmation of IPF. The presence of extensive mosaic attenuation, ground glass opacity, cysts and nodules suggest an alternative diagnosis (58). In the asymptomatic smoking population interstitial lung abnormalities (ILAs) will be detected. These ILAs are essentially very early fibrosis involving less than $5 \%$ of the lung parenchyma. Combined pulmonary fibrosis and emphysema is an important radiographic diagnosis with significant centrilobular type emphysema and a UIP pattern (59). Radiologists should describe these findings as they are associated with an increased risk of lung cancer and will likely progress over time (60).

Patients with fibrosis of the lung are living longer because of earlier interventions and treatment of co-morbidities. The longer patients live with fibrosis the greater is their risk for developing lung cancer. The lung cancer that occurs in the setting of pulmonary fibrosis is different from the lung cancer that occurs in emphysema as it typically occurs within the fibrosis and thus lower lobe predominant and peripheral, and the cancers are more aggressive (61).

Early findings of UIP have been classified as prehoneycomb and honeycomb (HC) findings $(56,62)$. Other interstitial diseases can also be identified and may differ in location and findings (62). Pre-honeycomb findings may start with traction bronchiectasis alone and then progress to ground-glass opacification and reticulations, typically at the periphery of the lungs and at the lung bases. The likelihood of disease progression is associated with honeycombing. Early identification is important for early treatment.

ILAs are minimal, incidentally identified parenchymal abnormalities on CT scan, affecting more than $5 \%$ of the lung. ILAs include traction bronchiectasis, ground-glass opacities, reticular abnormalities, and honeycombing. ILAs are associated with increased risk of developing fibrosis with its associated respiratory compromise and mortality (63). Whittaker Brown et al. (64) demonstrated that a significant number of smokers have ILAs and these smokers are at increased risk for lung cancer. People with ILAs should be screened for lung cancer and referred to pulmonologists for observation and/or early treatment (65).

\section{Mediastinal abnormalities}

\section{Mediastinal abnormalities and masses}

Mediastinal abnormalities and masses can occur anywhere in the mediastinum, including in the thymus, heart, and esophagus; and masses in the neck, such as the thyroid, may extend into the mediastinum (66). Such mediastinal and soft 
tissues masses are documented as to location and size. With the introduction of CT scanners, recognition of mediastinal abnormalities markedly increased, including vascular anomalies.

The frequency of mediastinal abnormalities in the context of LDCT screening for lung cancer, which focuses on older smokers, was not known. For this reason, in 2006, we published the results of our review of the LDCTs of 9,263 participants in I-ELCAP performed between 1992 and 2002 (66). We found that $71(0.77 \%)$ had a mediastinal mass on baseline screening. Of the 71 participants with mediastinal masses, 41 were thymic masses, 16 thyroid masses, two esophageal cancers, six tracheal-esophageal diverticula, and six were masses other than those listed. Among the 11,126 annual repeat screenings, only one $(0.01 \%)$ new mediastinal mass was identified (66). Of the 41 thymic masses, 5 were larger than $30 \mathrm{~mm}$ in diameter and all five were resected and the diagnosis was thymic carcinoma in one and noninvasive thymomas for the other four. Of the remaining 36 thymic masses, 25 had follow-up LDCT on year later; of the 25 , five increased, two decreased, and 18 remained unchanged. All 16 thyroid masses were due to goiter and remained unchanged on follow-up 1 year later.

\section{Thymic masses}

Thymic masses of screening participants were reviewed (66). Based on the frequency and natural course of thymic masses identified in baseline and annual repeat screenings for lung cancer, the following work-up recommendations are made: If the mass is $3.0 \mathrm{~cm}$ or less in diameter on baseline CT without invasive features (e.g., irregular borders or loss of fat planes), follow-up CT 1 year later is recommended. If the thymic mass is greater than $3.0 \mathrm{~cm}$ or shows growth on the follow-up CT, then further workup according to standard practice is recommended.

\section{Thyroid}

Thyroid abnormalities were evaluated in 2,309 participants who had baseline and annual repeat screening at Mount Sinai Health System under an IRB approved HIPAA compliant LDCT screening program between January 2010 to December 2016. This review identified thyroid nodules in $57(2.5 \%)$ participants on baseline screenings. Increasing age, increasing CAC score and increasing breast density grade were significant predictors for women having an incidental thyroid nodule (ITN). No significant predictors were found for men. New thyroid nodules were identified in 7 (0.15\%) participants among 4,792 of annual repeat LDCTs, suggesting slow growth as it would take approximately 16.8 years of growth on average for a new thyroid nodule detected on annual rounds of screening. The American College of Radiology (ACR) provides recommendations for ITNs detected in general population (67).

\section{Breast abnormalities}

\section{Breast density}

Breast density can be easily determined on LDCT (68). It is an important risk factor for breast cancer and may also mask tumors on mammography even when digital breast tomosynthesis is used (69). More than 30 states in the United States passed mandatory density notification to patients after their mammograms so that women are better informed about their breast cancer risk and can choose to have supplemental screening with breast ultrasound or MRI. In 2019, the FDA began the process of making reporting breast density on mammography a federal requirement in both the health provider report and the patient lay letter. For women who do not have mammograms, however, LDCT may be the first and perhaps only way a woman can learn about her breast density as it cannot be determined by physical exam.

Breast density is defined in the Breast Imaging Reporting and Data System (BI-RADS) Atlas developed by the ACR (70). The four BI-RADS breast categories are: (I) almost entirely fatty; (II) scattered fibroglandular densities; (III) heterogeneously dense (which may obscure small masses), and (IV) extremely dense.

The clinically relevant differentiation is between categories A-B and C-D (68,71). If the breast tissue is, category $\mathrm{C}$ or $\mathrm{D}$, this should be noted in the report as it suggests an increased risk for breast cancer and if clinically indicated, ultrasound (72) or MRI (73) of the breast may be considered for supplemental screening as the dense tissue might obscure an early cancer or precursor lesion on mammography.

\section{Breast masses}

Breast masses and larger breast calcifications can be seen on LDCT (74). Often these findings are known from prior breast imaging and require no work-up. Others are typically benign and also require no work-up, but if new or changing, 
a recommendation for dedicated breast imaging may be life changing. The breast findings on LDCT can be stratified into a system analogous to BI-RADS for ease of reporting and follow up (70).

Vascular calcifications seen on mammograms also provide information about coronary artery disease and should be reported $(75,76)$. This added benefit of mammography created much interest (77). As some suggested that this information did not need to be reported, a subsequent survey (78) showed that women overwhelmingly wanted to have this information if available from mammography.

\section{Abdominal abnormalities}

\section{Adrenal lesions}

Adrenal lesions may be due to diffuse enlargement, focal nodularity, or a mass $(79,80)$. The prevalence and natural history of adrenal lesions have been reported in many different settings. They increase with increasing age, from $0.2 \%$ of CT scans of people aged 20-29 years to $7-10 \%$ for older people (81). They are more frequently found in women, although this may be because women had abdominal imaging more frequently as no gender differences have been found in autopsy series (82). Reports suggested that $70-94 \%$ of the adrenal abnormalities are due to benign, non-secreting hyperplastic glands in asymptomatic people without history of known malignancy.

We determined the frequency of adrenal enlargement in 4,776 participants of CT screening for lung cancer at the Mount Sinai Health System who had signed HIPAAcompliant informed consents (83). We demonstrated the progression of enlargement during follow-up, separately for the baseline and annual repeat rounds. The adrenal gland was defined as enlarged when it measured $6 \mathrm{~mm}$ or more at its largest diameter.

On baseline screening, 202 (4\%) of 4,776 participants had adrenal enlargement. Significant factors were age $(\mathrm{OR}=1.4,95 \% \mathrm{CI}: 1.2-1.7)$ and current smoker $(\mathrm{OR}=1.8$, 95\% CI: 1.3-2.4). Frequency of adrenal enlargement increased with increasing pack-years of smoking $(\mathrm{P}=0.04)$. Follow-up scans 7-18 months after baseline for 133 of the 200 cases with adrenal enlargement less than $40 \mathrm{~mm}$ showed it decreased or was unchanged in $85(64 \%)$, and increased by less than $10 \mathrm{~mm}$ in 48 (36\%). Five $(0.04 \%)$ cases of adrenal enlargement were newly identified after baseline screening, but none increased beyond $40 \mathrm{~mm}$ on followup. Adrenal enlargement was a significant predictor of a subsequent diagnosis of lung cancer in screening $(\mathrm{OR}=2.0$, 95\% CI: 1.2-3.4).

We recommended that participants with adrenal enlargement of $40 \mathrm{~mm}$ or less in largest transverse diameter on baseline and repeat screening with low attenuation can be reasonably assessed on subsequent annual screening, based on our evaluation and that of others as described in the article (83). Suspicious imaging findings suggesting more immediate workup are: irregular borders, heterogeneity, hemorrhage, central necrosis, or calcifications. When either adrenal gland measures $40 \mathrm{~mm}$ or more in the largest transverse diameter, further evaluation is recommended (83).

\section{Hepatic steatosis $(H S)$}

HS is the most common finding in the upper abdomen in asymptomatic people. It is due to an excess accumulation of lipids in hepatocytes and can be progressive and lead to cirrhosis, liver failure, and hepatocellular carcinoma (84-86). Radiologists have an important role as this condition is frequently asymptomatic and cannot be diagnosed by any currently available blood test. It can be due to non-alcoholic and alcoholic liver diseases. Prevalence rates of $17-46 \%$ for HS have been reported in adults in Western countries (87). A single publication on follow-up liver assessment using magnetic resonance imaging reported that $13.8 \%$ of the 367 participants without known liver disease had HS (88). LDCT provides fast, reproducible, objective, and noninvasive measurements of moderate and severe HS (89-92).

Review of baseline LDCT scans of the chest of 170 participants in an IRB approved study between August 2011 and April 2016 was performed (93). The liver was divided into four sectors (left lateral, left medial, right anterior, right posterior), as defined by the Couinaud segmentation system. In each sector, a standard $1.0 \mathrm{~cm}^{2}$ region of interest (ROI) was selected, avoiding other lesions and large blood vessel. Measurements were made using standard mediastinal window settings (width $350 \mathrm{HU}$; level $25 \mathrm{HU}$ ) and the average attenuation and its standard deviation were calculated. Splenic CT attenuation measurement are obtained in the same fashion. Average liver attenuation was 57.6 HU (standard deviation of 9.3) and average liver/ spleen $(\mathrm{L} / \mathrm{S})$ ratio was 1.3 (SD 0.3). Liver attenuation was $<40 \mathrm{HU}$ for $9(5.3 \%)$, liver/spleen $(\mathrm{L} / \mathrm{S})$ ratio $<0.8$ for 6 (3.5\%) and either $<40 \mathrm{HU}$ or $\mathrm{L} / \mathrm{S}$ ratio $<0.8$ for $9(5.3 \%)$. Male sex $(\mathrm{P}=0.004)$, diabetes $(\mathrm{P}=0.0005)$, emphysema $(\mathrm{P}=0.03)$, and high $\mathrm{BMI}(\mathrm{P}=0.0006)$ were significant 
predictors of HS. Aspartate aminotransferase $(\mathrm{P}=0.0018)$ and alanine aminotransferase $(\mathrm{P}=0.012)$ were negatively correlated with liver attenuation. Thus, LDCT detected HS in asymptomatic participants with frequencies similar to previous reports $(94,95)$.

Based on the findings, if liver attenuation is below $40 \mathrm{HU}$ and/or the L/S ratio below 0.8 , we recommend follow-up with a primary care physician or liver specialist for further evaluation of possible hepatic steatosis detection $(93,96)$.

\section{Acknowledgments}

We thank the clinicians in cardiology (Dr. Joseph Shemesh), pulmonary medicine (Drs. Javier Zulueta, Larry Di Fabrizio, and David Steiger), liver diseases (Dr. Andrea Branch) who have worked with us to develop the recommendations.

Funding: None.

\section{Footnote}

Provenance and Peer Review: This article was commissioned by the Guest Editor (Witold Rzyman) for the series "Implementation of CT-based screening of lung cancer" published in Translational Lung Cancer Research. The article has undergone external peer review.

Conflicts of Interest: All authors have completed the ICMJE uniform disclosure form (available at http://dx.doi. org/10.21037/tlcr-20-746). The series "Implementation of CT-based screening of lung cancer" was commissioned by the editorial office without any funding or sponsorship. Dr. Salvatore reports grants and other from Genentech, grants and other from Boehringer Ingelheim, outside the submitted work. Dr. DFY reports other from Accumetra, other from GRAIL, outside the submitted work; In addition, Dr. DFY is a named inventor on a number of patents and patent applications relating to the evaluation of diseases of the chest including measurement of nodules. Some of these, which are owned by Cornell Research Foundation (CRF), are non-exclusively licensed to General Electric. As an inventor of these patents, he is entitled to a share of any compensation which CRF may receive from its commercialization of these patents. He is also an equity owner in Accumetra, a privately held technology company committed to improving the science and practice of image-based decision making. Dr. DFY also serves on the advisory board of GRAIL. Dr. CIH is a named inventor on a number of patents and patent applications relating to the evaluation of pulmonary nodules on CT scans of the chest which are owned by Cornell Research Foundation (CRF). Since 2009, Dr. CIH does not accept any financial benefit from these patents including royalties and any other proceeds related to the patents or patent applications owned by CRF. Dr. CIH is the President and serve on the board of the Early Diagnosis and Treatment Research Foundation. She receives no compensation from the Foundation. The Foundation is established to provide grants for projects, conferences, and public databases for research on early diagnosis and treatment of diseases. Recipients include, I-ELCAP, among others. The funding comes from a variety of sources including philanthropic donations, grants and contracts with agencies (federal and non-federal), imaging and pharmaceutical companies relating to image processing assessments. The various sources of funding exclude any funding from tobacco companies or tobacco-related sources. The other authors have no conflicts of interest to declare.

Ethical Statement: The authors are accountable for all aspects of the work in ensuring that questions related to the accuracy or integrity of any part of the work are appropriately investigated and resolved.

I-ELCAP Investigators: Mount Sinai School of Medicine, New York, NY: Claudia I. Henschke, Principal Investigator, David F. Yankelevitz, Rowena Yip, Artit Jirapatnakul, Raja Flores, Andrea Wolf; Weill Cornell Medical College: Daniel M. Libby, James P. Smith, Mark Pasmantier; Cornell University: A. P. Reeves; CBNS, City University of New York at Queens College, Queens, NY; Steven Markowitz, Albert Miller; Fundacion Instituto Valenciano de Oncologia, Valencia, Spain: Jose Cervera Deval; University of Toronto, Princess Margaret Hospital, Toronto, Canada: Heidi Roberts, Demetris Patsios; Azumi General Hospital, Nagano, Japan: Shusuke Sone, Takaomi Hanaoka; Clinica Universitaria de Navarra, Pamplona, Spain: Javier Zulueta, Juan P. de-Torres, Maria D. Lozano; Swedish Medical Center, Seattle, WA: Ralph Aye, Kristin Manning; Christiana Care, Helen F. Graham Cancer Center, Newark, DE: Thomas Bauer; National Cancer Institute Regina Elena, Rome, Italy: Stefano Canitano, Salvatore Giunta; St.Agnes Cancer Center, Baltimore, MD: Enser Cole; LungenZentrum Hirslanden, Zurich, Switzerland: Karl Klingler; Columbia University Medical Center, New York, NY: John H.M. Austin, Gregory D. N. Pearson; Hadassah Medical Organization, Jerusalem, Israel: Dorith Shaham; 
Holy Cross Hospital Cancer Institute, Silver Spring, MD: Cheryl Aylesworth; Nebraska Methodist Hospital, Omaha NE: Patrick Meyers; South Nassau Communities Hospital, Long Island, NY: Shahriyour Andaz; Eisenhower Lucy Curci Cancer Center, Rancho Mirage, CA; Davood Vafai; New York University Medical Center, New York, NY: David Naidich, Georgeann McGuinness; Dorothy E. Schneider Cancer Center, Mills-Peninsula Health Services, San Mateo, CA: Barry Sheppard; State University of New York at Stony Brook, Stony Brook, NY: Matthew Rifkin; ProHealth Care Regional Cancer Center, Waukesha \& Oconomowoc Memorial Hospitals, Oconomowoc, WI: M. Kristin Thorsen, Richard Hansen; Maimonides Medical Center, Brooklyn, NY: Samuel Kopel; Wellstar Health System, Marietta GA: William Mayfield; St. Joseph Health Center, St. Charles, MO: Dan Luedke; Roswell Park Cancer Institute, Buffalo, NY: Donald Klippenstein, Alan Litwin, Peter A. Loud; Upstate Medical Center, Syracuse, NY: Leslie J. Kohman, Ernest M. Scalzetti; Jackson Memorial Hospital, University of Miami, Miami, FL; Richard Thurer, Nestor Villamizar; State University of New York, North Shore-Long Island Jewish Health System, New Hyde Park, NY: Arfa Khan, Rakesh Shah; The $5^{\text {th }}$ Affiliated Hospital of Sun Yat-Sen University, Zhuhai, China: Xueguo Liu; Mercy Medical Center, Rockville Center, NY: Gary Herzog; Shin Kong Wu Ho-Su Memorial Hospital, Taipei, Taiwan: Diana Yeh; National Cancer Institute of China, Beijing, China: Ning Wu; Staten Island University Hospital, Staten Island NY: Joseph Lowry, Mary Salvatore; Central Main Medical Center: Carmine Frumiento; Mount Sinai School of Medicine, New York, NY: David S. Mendelson; Georgia Institute for Lung Cancer Research, Atlanta, GA: Michael V. Smith; The Valley Hospital Cancer Center, Paramus NJ: Robert Korst; Health Group Physimed/ McGill University, Montreal, CA: Jana Taylor; Memorial Sloan-Kettering Cancer Center, New York, NY: Michelle S. Ginsberg; John Muir Cancer Institute, Concord CA: Michaela Straznicka; Atlantic Health Morristown Memorial Hospital, Morristown NJ: Mark Widmann; Alta Bates Summit Medical Center, Berkeley CA: Gary Cecchi; New York Medical College, Valhalla, NY: Terence A.S. Matalon; St. Joseph's Hospital, Atlanta GA: Paul Scheinberg; Mount Sinai Comprehensive Cancer Center, Miami Beach, FL: Shari-Lynn Odzer; Aurora St. Luke's Medical Center, Milwaukee WI: David Olsen; City of Hope National Medical Center, Duarte, CA: Fred Grannis, Arnold Rotter; Evanston Northwestern Healthcare Medical Group, Evanston, IL: Daniel Ray; Greenwich Hospital, Greenwich,
CT: David Mullen; Our Lady of Mercy Medical Center, Bronx, NY: Peter H. Wiernik; Baylor University Medical Center, Dallas TX: Edson H. Cheung; Sequoia Hospital, Redwood City CA: Melissa Lim; Glens Falls Hospital, Glens Falls NY: Louis DeCunzo; Atlantic Medical Imaging, Atlantic City NJ: Robert Glassberg; Karmanos Cancer Institute, Detroit, MI: Harvey Pass, Carmen Endress; Rush University, Chicago IL: Mark Yoder, Palmi Shah; Building Trades, Oak Ridge TN: Laura Welch; Sharp Memorial Hospital, San Diego, CA: Michael Kalafer; Newark Beth Israel Medical Center, Newark NJ Jeremy Green; Guthrie Cancer Center, Sayre PA: James Walsh, David Bertsch; Comprehensive Cancer Centers of the Desert, Palm Springs CA: Elmer Camacho; Dickstein Cancer Treatment Center, White Plains Hospital, White Plains NY: Cynthia Chin; Presbyterian Healthcare, Charlotte NC: James O'Brien; University of Toledo, Toledo OH: James C. Willey.

Open Access Statement: This is an Open Access article distributed in accordance with the Creative Commons Attribution-NonCommercial-NoDerivs 4.0 International License (CC BY-NC-ND 4.0), which permits the noncommercial replication and distribution of the article with the strict proviso that no changes or edits are made and the original work is properly cited (including links to both the formal publication through the relevant DOI and the license). See: https://creativecommons.org/licenses/by-nc-nd/4.0/.

\section{References}

1. Centers of Medicare and Medicaid Services. Decision memo for screening for lung cancer with low dose computed tomography (LDCT) (CAG-00439N) [cited 2015 April 21]. Available online: http://www.cms.gov/ medicare-coverage-database/details/nca-decision-memo. aspx?NCAId.274

2. US Burden of Disease Collaborators, Mokdad AH, Ballestros K, et al. The State of US Health, 1990-2016: Burden of Diseases, Injuries, and Risk Factors Among US States. JAMA 2018;319:1444-72.

3. World Health Organization. Global Health Estimates 2016: Deaths by Cause, Age, Sex, by Country and by Region, 2000-2016. Geneva, 2018.

4. Shemesh J, Henschke CI, Farooqi A, Yip R, Yankelevitz DF, Shaham D, Miettinen OS. Frequency of coronary artery calcification on low-dose computed tomography screening for lung cancer. Clin Imaging 2006;30:181-5.

5. Shemesh J, Henschke CI, Shaham D, et al. Ordinal 
Scoring of Coronary Artery Calcifications on Low-

Dose CT Scans of the Chest is Predictive of Death from

Cardiovascular Disease. Radiology 2010;257:541-8.

6. de Torres JP, Bastarrika G, Wisnivesky JP, et al.

Assessing the relationship between lung cancer risk and emphysema detected on low-dose CT of the chest. Chest 2007;132:1932-8.

7. Zulueta JJ, Wisnivesky JP, Henschke CI, et al. Emphysema scores predict death from COPD and lung cancer. Chest 2012;141:1216-23.

8. Lee CI, Forman HP. What we can and cannot see coming. Radiology 2010;257:313-4.

9. Budoff MJ, Hamirani YS, Gao YL, et al. Measurement of thoracic bone mineral density with quantitative CT. Radiology 2010;257:434-40.

10. Gondrie MJ, Mali WP, Jacobs PC, et al. Cardiovascular disease: prediction with ancillary aortic findings on chest CT scans in routine practice. Radiology 2010;257:549-59.

11. Steiger D, Filopei J, Siddiqi M, et al. Evidence Of Emphysema In A Cohort Of Participants Without Symptoms Undergoing Low Dose Chest CT Screening For Lung Cancer. Am J Respir Crit Care Med 2017;195:1.

12. Budoff MJ, Nasir K, Katz R, et al. Thoracic aortic calcification and coronary heart disease events: the multiethnic study of atherosclerosis (MESA). Atherosclerosis 2011;215:196-202.

13. Hecht HS, Cronin P, Blaha MJ, et al. 2016 SCCT/ STR guidelines for coronary artery calcium scoring of noncontrast noncardiac chest CT scans: A report of the Society of Cardiovascular Computed Tomography and Society of Thoracic Radiology. J Cardiovasc Comput Tomogr 2017;11:74-84.

14. Hanna WC, Paul NS, Darling GE, et al. Minimal-dose computed tomography is superior to chest $\mathrm{x}$-ray for the follow-up and treatment of patients with resected lung cancer. J Thorac Cardiovasc Surg 2014;147:30-3.

15. Schaal M, Severac F, Labani A, et al. Diagnostic Performance of Ultra-Low-Dose Computed Tomography for Detecting Asbestos-Related Pleuropulmonary Diseases: Prospective Study in a Screening Setting. PLoS One 2016;11:e0168979.

16. van den Berk IA, Kanglie MM, van Engelen TS, et al. OPTimal IMAging strategy in patients suspected of nontraumatic pulmonary disease at the emergency department: chest X-ray or ultra-low-dose CT (OPTIMACT)—a randomised controlled trial chest X-ray or ultra-low-dose CT at the ED: design and rationale. Diagn Progn Res 2018;2:20.
17. Kroft LJ, van der Velden L, Girón IH, et al. Added Value of Ultra-low-dose Computed Tomography, Dose Equivalent to Chest X-Ray Radiography, for Diagnosing Chest Pathology. J Thorac Imaging 2019;34:179.

18. Henschke CI, McCauley D, Yankelevitz D, et al. Early Lung Cancer Action Project: overall design and findings from baseline screening. Lancet 1999;354:99-105.

19. International Early Lung Cancer Action Program Investigators, Henschke CI, Yankelevitz DF, et al. Survival of patients with stage I lung cancer detected on CT screening. N Engl J Med 2006;355:1763-71.

20. Yip R, Henschke CI, Yankelevitz DF, et al. The impact of the regimen of screening on lung cancer cure: a comparison of I-ELCAP and NLST. Eur J Cancer Prev 2015;24:201-8.

21. International Early Lung Cancer Action Program Investigators. International Early Lung Cancer Action Program protocol [cited 2018 Jan 3rd]. Available online: www.IELCAP.org/protocols

22. Mini Symposium on "Lung Cancer Screening, Opportunistic Evaluation of Findings". IASLC 2019 WCLC World Conference on Lung Cancer; 2019 September 9, 2019; Barcelona, Spain.

23. Xie Y, Liu S, Miller A, et al. Coronary artery calcification identification and labeling in low-dose chest CT images. Medical Imaging 2017: Computer-Aided Diagnosis; 2017; Orlando, FL: International Society for Optics and Photonics.

24. Fuhrman JD, Crosby J, Yip R, et al. Detection and classification of coronary artery calcifications in low dose thoracic CT using deep learning. Medical Imaging 2019: Computer-Aided Diagnosis; 2019: International Society for Optics and Photonics.

25. Fuhrman J, Yip R, Henschke C, et al. Cascade of U-Nets in the detection and classification of coronary artery calcium in thoracic low-dose CT. SPIE: Medical Imaging 2020 February; Houston, TX.

26. Lessmann N, van Ginneken B, Zreik M, et al. Automatic Calcium Scoring in Low-Dose Chest CT Using Deep Neural Networks With Dilated Convolutions. IEEE Trans Med Imaging 2018;37:615-25.

27. Xie Y, Padgett J, Biancardi AM, et al. Automated aorta segmentation in low-dose chest CT images. Int J Comput Assist Radiol Surg 2014;9:211-9.

28. Gierada DS, Guniganti P, Newman BJ, et al. Quantitative CT Assessment of Emphysema and Airways in Relation to Lung Cancer Risk. Radiology 2011;261:950-9.

29. Keller BM, Reeves AP, Henschke CI, et al. Multivariate 
compensation of quantitative pulmonary emphysema metric variation from low-dose, whole-lung CT scans. AJR Am J Roentgenol 2011;197:W495-502.

30. Raghunath S, Rajagopalan S, Karwoski RA, et al. Quantitative stratification of diffuse parenchymal lung diseases. PLoS One 2014;9:e93229.

31. Humphries SM, Notary AM, Centeno JP, et al. Deep Learning Enables Automatic Classification of Emphysema Pattern at CT. Radiology 2020;294:434-44.

32. J L, AP R. Segmentation of the airway tree from chest CT using local volume of interest. Second International Workshop of Pulmonary Image Analysis. 2009:333-40.

33. Lee J, Reeves AP, Fotin S, et al. Human airway measurement from CT images. SPIE International Symposium on Medical Imaging; 2008.

34. Matsuoka S, Yamashiro T, Washko GR, et al. Quantitative CT assessment of chronic obstructive pulmonary disease. Radiographics 2010;30:55-66.

35. Kim SS, Seo JB, Lee HY, et al. Chronic obstructive pulmonary disease: lobe-based visual assessment of volumetric CT by Using standard images--comparison with quantitative CT and pulmonary function test in the COPDGene study. Radiology 2013;266:626-35.

36. Xie Y, Salvatore M, Liu S, et al. Identification of earlystage usual interstitial pneumonia from low-dose chest CT scans using fractional high-density lung distribution. Medical Imaging 2017: Computer-Aided Diagnosis; 2017: International Society for Optics and Photonics.

37. van Royen FS, Moll SA, van Laar JM, et al. Automated CT quantification methods for the assessment of interstitial lung disease in collagen vascular diseases: A systematic review. Eur J Radiol 2019;112:200-6.

38. Liu S, Margolies L, Xie Y, et al. Fully automated breast density assessment from low-dose chest CT. SPIE Medical Imaging; 14 February 2017.

39. Xie Y, Liang M, Yankelevitz DF, et al. Automated segmentation of cardiac visceral fat in low-dose noncontrast chest CT images. SPIE Medical Imaging; 2015.

40. Jirapatnakul A, Reeves AP, Lewis S, et al. Automated measurement of liver attenuation to identify moderate-tosevere hepatic steatosis from chest CT scans. Eur J Radiol 2020;122:108723.

41. Henschke CI, Yip R, Shaham D, et al. The Regimen of Computed Tomography Screening for Lung Cancer. Lessons Learned Over 25 Years From the International Early Lung Cancer Action Program. J Thorac Imaging 2021;36:6-23.

42. Owens DS, Budoff MJ, Katz R, et al. Aortic valve calcium independently predicts coronary and cardiovascular events in a primary prevention population. JACC Cardiovasc Imaging 2012;5:619-25.

43. Blaha MJ, Budoff MJ, Rivera JJ, et al. Relation of aortic valve calcium detected by cardiac computed tomography to all-cause mortality. Am J Cardiol 2010;106:1787-91.

44. Pradelli D, Faden G, Mureddu G, et al. Impact of aortic or mitral valve sclerosis and calcification on cardiovascular events and mortality: a meta-analysis. Int J Cardiol 2013;170:e51-5.

45. Coffey S, Cox B, Williams MJ. The prevalence, incidence, progression, and risks of aortic valve sclerosis: a systematic review and meta-analysis. J Am Coll Cardiol 2014;63:2852-61.

46. Zhu Y, Wang Y, Gioia W, et al. Visual Scoring of Aortic Valve Calcifications on Low-Dose CT in Lung Cancer Screening. Eur Radiol 2020;30:2658-68.

47. Zhu Y, Yip R, Shemesh J, et al. Combined aortic valve and coronary artery calcifications on low-dose CT in lung cancer screening for predicating cardiovascular death. Submitted.2019.

48. Sims MW, Margolis DJ, Localio AR, et al. Impact of pulmonary artery pressure on exercise function in severe COPD. Chest 2009;136:412-9.

49. Hurdman J, Condliffe R, Elliot CA, et al. Pulmonary hypertension in COPD: results from the ASPIRE registry. Eur Respir J 2013;41:1292-301.

50. Wells JM, Washko GR, Han MK, et al. Pulmonary arterial enlargement and acute exacerbations of COPD. N Engl J Med 2012;367:913-21.

51. Lettieri CJ, Nathan SD, Barnett SD, Ahmad S, Shorr AF. Prevalence and outcomes of pulmonary arterial hypertension in advanced idiopathic pulmonary fibrosis. Chest 2006;129:746-52.

52. Ghio S, Gavazzi A, Campana C, et al. Independent and additive prognostic value of right ventricular systolic function and pulmonary artery pressure in patients with chronic heart failure. J Am Coll Cardiol 2001;37:183-8.

53. Mathai SC, Ryan JJ. The Growing Burden of Pulmonary Hypertension in the Modern Era: A Zebra No More? Circ Cardiovasc Qual Outcomes 2018;11:e004536.

54. Steiger D, Han D, Yip R, et al. Increased main pulmonary artery diameter and main pulmonary artery to ascending aortic diameter ratio in smokers undergoing lung cancer screening. Clin Imaging 2020;63:16-23.

55. Aberle DR, Adams A, Berg C, et al. Reduced lung-cancer mortality with low-dose computed tomographic screening. N Engl J Med 2011;365:395-409. 
56. Salvatore M, Henschke C, Yip R, et al. Evidence of Interstitial Lung Disease on Low-Dose Chest CT Images: Prevalence, Patterns, and Progression. AJR Am J Roentgenol 2016;206:487-94.

57. Raghu G, Collard HR, Egan JJ, et al. An Official ATS/ ERS/JRS/ALAT Statement: Idiopathic pulmonary fibrosis: Evidence-based guidelines for diagnosis and management. Am J Respir Crit Care Med 2011;183:788-824.

58. Lynch DA, Sverzellati N, Travis WD, et al. Diagnostic criteria for idiopathic pulmonary fibrosis: a Fleischner Society White Paper. Lancet Respir Med 2018;6:138-53.

59. Cottin V, Nunes H, Brillet PY, et al. Combined pulmonary fibrosis and emphysema: a distinct underrecognised entity. Eur Respir J 2005;26:586-93.

60. Salvatore M, Singh A, Yip R, et al. Progression of probable UIP and UIP on HRCT. Clin Imaging 2019;58:140-4.

61. Pagano A, Siddique M, Chung M, et al. editors. Volume Doubling Time of Primary Lung Cancers in Interstitial Lung Disease. Radiological Society of North America 2019 Scientific Assembly and Annual Meeting; 2019

December 1 - December 6, 2019; Chicago, IL.

62. Southern BD, Scheraga RG, Yadav R. Managing interstitial lung disease detected on CT during lung cancer screening. Cleve Clin J Med 2016;83:55-65.

63. Tzilas V, Bouros D. Interstitial Lung Abnormalities: A Word of Caution. Chest 2019;156:1037-8.

64. Whittaker Brown SA, Padilla M, Mhango G, et al. Interstitial Lung Abnormalities and Lung Cancer Risk in the National Lung Screening Trial. Chest 2019;156:1195-203.

65. Ash SY, Washko GR. Interstitial lung abnormalities: risk and opportunity. Lancet Respir Med 2017;5:95-6.

66. Henschke CI, Lee IJ, Wu N, et al. CT screening for lung cancer: Prevalence and incidence of mediastinal masses. Radiology 2006;239:586-90.

67. Hoang JK, Langer JE, Middleton WD, et al. Managing Incidental Thyroid Nodules Detected on Imaging: White Paper of the ACR Incidental Thyroid Findings Committee. J Am Coll Radiol 2015;12:143-50.

68. Salvatore M, Margolies L, Kale M, et al. Breast density: comparison of chest CT with mammography. Radiology 2014;270:67-73.

69. Kontos D, Bakic PR, Carton AK, et al. Parenchymal Texture Analysis in Digital Breast Tomosynthesis for Breast Cancer Risk Estimation: A Preliminary Study. Acad Radiol 2009;16:283-98.

70. Sickles E, D’Orsi C, Bassett L, et al. ACR BI-RADS® Mammography. Reston, VA: American College of
Radiology; 2013.

71. Margolies L, Salvatore M, Eber C, et al. The general radiologist's role in breast cancer risk assessment: breast density measurement on chest CT. Clinical Imaging 2015;39:979-82.

72. Mendelson E, Böhm-Vélez M, Berg W, et al. ACR BIRADS® Ultrasound. Reston, VA: American College of Radiology, 2013.

73. Morris E, Comstock C, Lee C, et al. ACR BI-RADS® Magnetic Resonance Imaging. Reston, VA2013.

74. Margolies LR, Salvatore M, Yip R, et al. The chest radiologist's role in invasive breast cancer detection. Clinical Imaging 2018;50:13-9.

75. Xie Y, Cham MD, Henschke C, et al. editors. Automated coronary artery calcification detection on low-dose chest CT images. SPIE International Symposium on Medical Imaging; 2014.

76. Margolies L, Salvatore M, Hecht HS, et al. Digital Mammography and Screening for Coronary Artery Disease. JACC Cardiovasc Imaging 2016;9:350-60.

77. Margolies LR, Yankelevitz DF, Henschke CI. Reply to "Mammographic Reporting of Breast Arterial Calcification". AJR Am J Roentgenol 2019;213:W146.

78. Margolies LR, Yip R, Hwang E, et al. Breast Arterial Calcification in the Mammogram Report: The Patient Perspective. AJR Am J Roentgenol 2019;212:209-14.

79. Vincent JM, Morrison I, Armstrong P, et al. The size of normal adrenal glands on computed tomography. Clin Radiol 1994;49:453-5.

80. Benitah N, Yeh BM, Qayyum A, et al. Minor morphologic abnormalities of adrenal glands at CT: prognostic importance in patients with lung cancer. Radiology 2005;235:517-22.

81. Boland GW, Blake MA, Hahn PF, et al. Incidental adrenal lesions: principles, techniques, and algorithms for imaging characterization. Radiology 2008;249:756-75.

82. Barzon L, Sonino N, Fallo F, et al. Prevalence and natural history of adrenal incidentalomas. Eur J Endocrinol 2003;149:273-85.

83. Hu M, Yip R, Yankelevitz DY, et al. CT screening for lung cancer: Frequency of enlarged adrenal glands identified in baseline and annual repeat rounds. Eur Radiol 2016;26:4475-81.

84. Bedossa P; FLIP Pathology Consortium. Utility and appropriateness of the fatty liver inhibition of progression (FLIP) algorithm and steatosis, activity, and fibrosis (SAF) score in the evaluation of biopsies of nonalcoholic fatty liver disease. Hepatology 2014;60:565-75. 
85. Angulo P, Kleiner DE, Dam-Larsen S, et al. Liver fibrosis, but no other histologic features, is associated with longterm outcomes of patients with nonalcoholic fatty liver disease. Gastroenterology 2015;149:389-97.e10.

86. Alvarez CS, Graubard BI, Thistle JE, et al. Attributable Fractions of NAFLD for Mortality in the United States: Results From NHANES III With 27 Years of Follow-up. Hepatology 2020;72:430-40.

87. European Association for the Study of the Liver (EASL); European Association for the Study of Diabetes (EASD); European Association for the Study of Obesity (EASO). EASL-EASD-EASO Clinical Practice Guidelines for the management of non-alcoholic fatty liver disease. J Hepatol 2016;64:1388-402.

88. Wong VW, Wong GL, Yeung DK, et al. Incidence of nonalcoholic fatty liver disease in Hong Kong: A population study with paired proton-magnetic resonance spectroscopy. J Hepatol 2015;62:182-9.

89. Davidson LE, Kuk JL, Church TS, Ross R. Protocol for measurement of liver fat by computed tomography. J Appl Physiol (1985) 2006;100:864-8.

90. Park SH, Kim PN, Kim KW, et al. Macrovesicular hepatic steatosis in living liver donors: use of CT for quantitative

Cite this article as: Yip R, Jirapatnakul A, Hu M, Chen X, Han D, Ma T, Zhu Y, Salvatore MM, Margolies LR, Yankelevitz DF, Henschke CI; for the I-ELCAP Investigators. Added benefits of early detection of other diseases on low-dose CT screening. Transl Lung Cancer Res 2021;10(2):1141-1153. doi: 10.21037/tlcr20-746 and qualitative assessment. Radiology 2006;239:105-12.

91. Kodama Y, Ng CS, Wu TT, et al. Comparison of CT methods for determining the fat content of the liver. AJR Am J Roentgenol 2007;188:1307-12.

92. Lee JY, Kim KM, Lee SG, et al. Prevalence and risk factors of non-alcoholic fatty liver disease in potential living liver donors in Korea: a review of 589 consecutive liver biopsies in a single center. J Hepatol 2007;47:239-44.

93. Chen X, Li K, Yip R, et al. Hepatic steatosis in participants in a program of low-dose CT screening for lung cancer. Eur J Radiol 2017;94:174-9.

94. Boyce CJ, Pickhardt PJ, Kim DH, et al. Hepatic steatosis (fatty liver disease) in asymptomatic adults identified by unenhanced low-dose CT. AJR Am J Roentgenol 2010;194:623-8.

95. Zeb I, Li D, Nasir K, et al. Computed tomography scans in the evaluation of fatty liver disease in a population based study: the multi-ethnic study of atherosclerosis. Acad Radiol 2012;19:811-8.

96. Dinani AM, Lewis S, Branch AD, et al. Working Up an Incidental Finding of Hepatic Steatosis on Imaging. Clin Liver Dis (Hoboken) 2020;16:58-62. 


\section{Appendix 1 LDCT findings and recommendations}

\section{Ordinal coronary artery calcification scoring}

Each coronary artery is identified (left main, left anterior descending, circumflex, and right coronary artery). Evidence of calcification in each artery is documented as none, minimal, moderate, or severe, scored as $0,1,2$, and 3 , respectively. Minimal calcification was defined if less than $1 / 3$ of the length of the entire artery, moderate as $1 / 3-2 / 3$, and severe as more than $2 / 3$ shows calcification. With 4 arteries thus scored, each person receives an Ordinal coronary artery calcium (CAC) Score in the range from 0 to 12. With additional effort, the Agatston, volume or mass calcium scores on LDCTs can also be obtained. New rapid scanning techniques minimize cardiac motion and allow for improved Agatston scoring on non-gated examinations.

\begin{tabular}{lll}
\hline Ordinal CAC score & Agatston score & Recommendation \\
\hline 0 & 0 & Probability of cardiovascular heart disease (CHD) is low. Reassure and keep healthy lifestyle \\
$1-3$ & $1-100$ & $\begin{array}{l}\text { Probability of CHD is mild to moderately increased; Recommend healthy lifestyle, moderate } \\
\text { statin, ASA }\end{array}$ \\
& $>100$ & $\begin{array}{l}\text { Probability of CHD is moderate to high. Healthy lifestyle; very intensive statin + second drug as } \\
\text { needed; ASA; consider function testing to r/o obstruction; aggressive BP lowering; referral to } \\
\text { internist or preventive cardiologist }\end{array}$ \\
& &
\end{tabular}

\section{Aortic valve calcifications}

Using standard mediastinal window setting (width and level of 350 and $50 \mathrm{HU}$ with $2.5 \mathrm{~mm}$ or $3.0 \mathrm{~mm}$ slice thickness) and if needed, multiplanar reconstruction to determine the location of calcifications. The extent of AVC was classified as:

* Mild: single or multiple isolated aortic valve calcifications;

* Moderate: multiple larger aortic valve calcifications, but not involving all three aortic leaflets;

* Severe: multiple larger aortic valve calcifications of all three aortic leaflets.

For moderate and severe AVC, recommend referral to a cardiologist is recommended and possible echocardiography, as there is a high probability of aortic stenosis (AS).

\section{Pulmonary artery hypertension}

The diameters of the main pulmonary artery (MPA) and ascending aorta (AA) are measured on an axial CT image at the level of the MPA bifurcation at the widest diameter vertical to its long axis and of the adjacent AA diameter.

If MPA $\geq 34 \mathrm{~mm}$ or MPA:AA $\geq 1.0$, recommend a pulmonary consult to determine its etiology and possible echo sonography.

\section{Emphysema}

The extent of emphysema is identified and classified as none, mild, moderate, or severe. Each subject receives an emphysema score in the range from 0 to 3 (7).

* Mild emphysema (Score 1): no discrete areas of decreased CT attenuation but splaying of blood vessels suggesting parenchymal expansion or having occasional discrete areas of decreased attenuation;

* Moderate emphysema (Score 2): discrete areas of decreased attenuation can be identified involving less than half of the lung parenchyma; and,

* Severe emphysema (Score 3): discrete areas of decreased attenuation can be identified involving more than half of the lung parenchyma.

If emphysema is present and previously unrecognized, recommend consultation with a pulmonologist.

\section{Interstitial findings}

Early findings of usual interstitial pneumonitis (UIP) include pre-honeycomb and honeycomb (HC) findings. Other interstitial diseases can also be identified and may differ in location and findings. Pre-honeycomb findings may start with traction bronchiectasis alone and then progress to ground-glass opacification and reticulations, typically at the periphery of 
the lungs and at the lung bases. The likelihood of disease progression is associated with honeycombing. Early identification is important and consultation with a pulmonologist is recommended.

If any of these findings are identified, recommend consultation with a pulmonologist.

\section{Mediastinal abnormalities}

Mediastinal masses can occur anywhere in the mediastinum, including in the thymus, heart, and esophagus; and masses in the neck, such as the thyroid, may extend into the mediastinum. Such mediastinal and soft tissues masses are documented as to location and size.

Thymic mass:

(I) $\leq 30 \mathrm{~mm}$ in diameter on baseline CT without invasive features (e.g., irregular borders or loss of fat planes), recommend follow-up CT in 1 year;

(II) $>30 \mathrm{~mm}$, recommend further workup according to standard practice is recommended.

Thyroid nodule:

(I) $\quad<15 \mathrm{~mm}$ on baseline or annual repeat LDCT with low HU attenuation, recommend annual follow-up;

(II) $<15 \mathrm{~mm}$ with heterogeneous enlarged appearance, recommend dedicated thyroid ultrasound examination;

(III) $\geq 15 \mathrm{~mm}$, recommend dedicated thyroid ultrasound examination.

\section{Breast density}

Using mediastinal settings, the CT images of the breast are reviewed and classified according to the Breast Imaging Reporting and Data System (BI-RADS) developed by the American College of Radiology (70). The BI-RADS classification identifies 4 grades according to the breast density. Calcifications seen in the breast also provide information about coronary artery disease and should be reported $(76,78)$.

The key differentiation is between Grades A-B and C-D $(68,71)$.

Grade A: almost entirely fatty

Grade B: there are scattered fibroglandular densities

Grade C: breasts are heterogeneously dense, which may obscure small masses

Grade D: breasts are extremely dense, which lowers the sensitivity of mammography

For Grade $\mathrm{C}$ or $\mathrm{D}$, recommend including this information in the report as it suggests an increased risk for breast cancer and if clinically indicated ultrasound (72) or MRI (73) of the breast is suggested instead of a mammogram as it might obscure an early cancer or precursor lesion.

\section{Adrenal glands}

Adrenal glands are measured on axial CT images. If the largest transverse diameter is: $\geq 40 \mathrm{~mm}$, recommend further evaluation according to standard of care;

$<40 \mathrm{~mm}$ and low attenuation (less than $10 \mathrm{HU}$ ), recommend annual low-dose CT scans to assess growth, but if the borders are irregular, heterogeneous, hemorrhagic, central necrosis or calcifications, recommend further evaluation.

\section{Liver steatosis}

The hepatic portal level is selected to measure liver attenuation $(\mathrm{HU})$ and the liver is divided into four sectors (left lateral, left medial, right anterior, right posterior). In each sector, a standard $1.0 \mathrm{~cm} 2$ region of interest (ROI) is selected, avoiding other lesions and large blood vessel. HU measurements are made using standard mediastinal window settings (width 350 $\mathrm{HU}$; level $25 \mathrm{HU}$ ) and the average attenuation and its standard deviation (SD) are calculated. Splenic average CT attenuation measurement are obtained in the same fashion in the upper, middle, and lower thirds of the spleen.

If the liver attenuation measurement $<40 \mathrm{HU}$ or liver-spleen ratio $<0.8$, recommend follow-up with a primary care physician or liver specialist for further evaluation. 